\title{
Intranasal Drug Delivery: Novel Delivery Route for Management of Parkinson's and Depression Neurological Disorders
}

\author{
Rekha Hondadakatti ${ }^{1}{ }^{(\mathbb{D})}$, Harshada R. Khandelwal ${ }^{2(\mathbb{D})}$, Anil K. Pawar ${ }^{2}{ }^{(\mathbb{D}}$, Gajanan S. Sanap ${ }^{3(\mathbb{D})}$, Sachin \\ D. Shinde ${ }^{4, * \text { (iD }}$ \\ 1 S.E.T's College of Pharmacy, S. R. Nagar, Dharwad, India-580002; rekha2003h@gmail.com (R.H.); \\ 2 R. C. Patel Institute of Pharmaceutical Education and Research, Dist-Dhule, Shirpur, Maharashtra, India 425405; \\ harshukhandelwal69@gmail.com (H.R.K.), anilpawar23@gmail.com (A.K.P.); \\ 3 Late Bhagirathi Yashvwantrao Pathrikar College of Pharmacy, Phulambri, Maharashtra, India-431111; \\ fourh29@gmail.com (G.S.S.); \\ 4 Shri R. D. Bhakt College of Pharmacy, Jalna, Maharashtra India-431203; sdshinde8390@gmail.com (S.D.S.); \\ * Correspondence: sdshinde8390@gmail.com (S.D.S.);
}

Received: 24.04.2021; Revised: 25.06.2021; Accepted: 28.06.2021; Published: 6.09.2021

\begin{abstract}
Neurological disorders are increasing worldwide due to the rapidly aging population, which increases healthcare costs. Drug delivery to the brain is challenging because of the brain's anatomy, and orally administered drugsare mostly unable to cross BBB. Intranasal (Nose to Brain) administration of drugs is one novel approach to address this challenge. Intranasal delivery has appeared to evade the blood-brain barrier (BBB) and deliver the drug into the CNS at a higher rate and degree than another traditional route. Transport of drugs from the nasal cavity to the brain along with olfactory and trigeminal nerves. The purpose of this review is drug delivery by the intranasal route for treating neurological disorders like Parkinson's and depression because drug delivery by other routes is unable to cross BBB. Still, delivery through the intranasal route by using the nanotechnology approach is possible to deliver the drug directly to CNS.
\end{abstract}

Keywords: intranasal; Parkinson's; depression; nanocarrier; nose to the brain.

(C) 2021 by the authors. This article is an open-access article distributed under the terms and conditions of the Creative Commons Attribution (CC BY) license (https://creativecommons.org/licenses/by/4.0/).

\section{Introduction}

The nasal pathway is a non-invasive administration route of active pharmaceutical ingredients for local, systemic, and CNS action. Intranasal drug delivery is a very conventional route for drug delivery from nose to the brain to treat neurological disorders [1]. Neurodegenerative disorder (ND) is a term used for conditions that affect the nervous system. ND results in damage to nerve cells of the nervous system, and damage causes chronic, progressive loss of neurons which causes decreasing cognitive abilities of the brain [2]. Nerve cells regulate our bodies with a response. Most degenerative nerve diseases are like Parkinson's, Alzheimer's disease, Depression, Huntington's disease are the most propagated diseases in the elderly population of developed and developing countries, and most are not easily cure. All of these have similar cellular progression; they present with different clinical features. For example, Huntington disease the main cause of this disease is a genetic modification that leads to severe motor restriction and eventually death [3]. Another one is Parkinson's disease due to the depletion of dopamine at the brain's basal ganglia, which is 
responsible for stiffness, rigidity, and tremors in the body's major muscles [4]. It is challenging for drug delivery to the central nervous system (CNS) because of the brain's anatomy [5]. The various types are drugs discovered for the treatment of CNS disease, but the clinical potency of any therapeutic agent is not only dependent on its bioavailability its depend on its ability to penetrate the protective layer, i.e., cerebrospinal fluid (CSF) and blood-brain barrier (BBB) which are the major obstacles for therapeutic agents which are used in the treatment of CNS because they restrict the entry of hydrophilic and large lipophilic molecules to the brain [6]. Therefore, the physicochemical properties of active therapeutic molecules and formulation for brain drug delivery are considered while designing any drug-delivering mode. Various techniques like intravenous, intrathecal, intraparenchymal are used and chemical modification, prodrug approach or antibody are used to increase drug targeting to CNS. Nose to Brain drug delivery has gained the interest of researchers as a potential delivery route for targeting the brain because of the Linkage between brain and nose by the olfactory route and by peripheral circulation [7]. The nose is the only organ for direct contact with the brain. Absorption of the molecules through the trigeminal and olfactory pathways from the nasal cavity provides a direct entrance to the brain, which executes the therapeutic effect of CNS-acting drugs [8]. This enhances therapeutic efficacy by circumventing hepatic circulation and reduces the systematic toxicity of centrally acting drugs. The nasal route has several advantages over oral or intravenous routes, including self-administration, shorter time onset of action, avoidance of hepatic first-pass metabolism, and bypassing the BBB may potentially increase the central nervous system (CNS) availability of the drug [9].

\subsection{Anatomy of the nasal cavity.}

In humans and animals function of the nasal cavity is not only breathing but also offers a protective function by filtering the air before reaching terminal alveoli; the other function is greater permeation from nasal mucosa [10]. The oral cavity and nasal cavity are separated from each other by the palatine bone. The total area of both nasal cavities is $\sim 150-160 \mathrm{~cm}^{2}$ [11]. The respiratory, olfactory, and vestibular regions are different parts of the nasal cavity involved in the different delivery routes of drug molecules. The first region is vestibular; it is most anterior and located immediately at the nostril opening; its surface area is around $0.6 \mathrm{~cm}^{2}$ and contains nasal hairs that filter inhaled particles. This region has the largest area at $\sim 130 \mathrm{~cm}^{2}$. The respiratory region covers the lateral walls of the nasal cavities, including the three projecting turbinates (lower, middle, and upper nasal turbinates). The other is the olfactory region; in the olfactory region, the olfactory bulb and nerves are responsible for olfaction function through neuroepithelium cells [12].

\subsection{Pathways for intranasal drug delivery.}

Based on current research, the pathways for the nose to brain drug delivery are olfactory neuronal pathways and trigeminal nerve pathways; the other is pathways involving CSF, vasculature, or lymphatic system [13]. Intranasal drug delivery may occur by either one or combined pathways (Figure 1). 


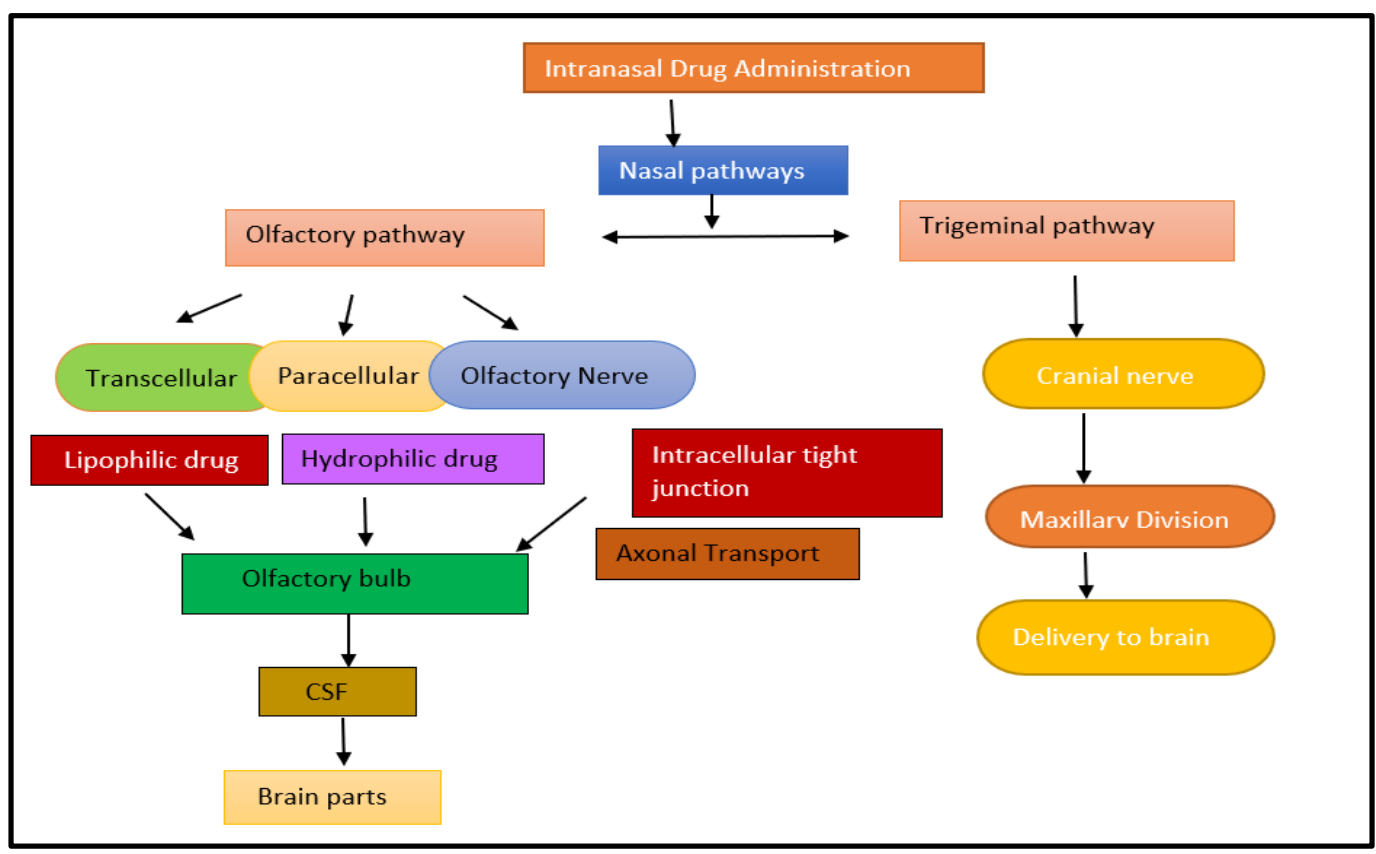

Figure 1. Pathways for intranasal drug delivery.

\subsubsection{Olfactory neuronal pathways.}

These are the most prevailing pathways of intranasal drug absorption to the brain; the delivered drug needs to cross the olfactory epithelium consumed by olfactory neurons. The movement is managed in three ways, paracellular movement or endocytosis and passive diffusion by neuronal cells. Lipophilic drugs are transported by passive diffusion, where paracellular movement is liable for the transportation of hydrophilic drugs. In this mechanism, the molecular weight and lipophilicity of the drug are important [14].

\subsubsection{Trigeminal nerves pathways.}

The trigeminal nerve is the largest cranial nerve, the trigeminal nerve passes on tactile data from the nasal cavity, the oral hole, the eyelids, and the cornea, to the CNS using the ophthalmic division (V1), the maxillary division (V2), or the mandibular division (V3) of the nerve $[12,15]$. It innervates the nasal olfactory epithelium toward one side, while the opposite end arrives at the cerebrum by two various destinations, close to the pons and cerebrum of the mind, just as to the frontal mind and olfactory bulb less significantly [9]. Accordingly, possible locales of medication conveyance to the cerebrum can likewise be benefited through trigeminal nerve pathways. Investigates indicated proof on the transport of insulin-like- growth factor-I to the CNS through intranasal organization through the olfactory and trigeminal pathways $[16,17]$.

\subsubsection{Cerebrospinal fluid and lymphatic pathways.}

This CSF and lymphatic pathways are connected from the CSF of subarachnoid space in the brain to the nasal lymphatic framework using olfactory nerves enveloped into perineurial space[18]. These pathways can transport a drug into the nasal cavity to the CSF and perivascular part to distribute to the other parts of the brain. The transport and distribution of drugs into CSF is depended on lipophilicity, molecular weight, and degree of ionization of the drug [19]. 


\section{Nanocarrier for Intranasal Drug Delivery}

\subsection{Lipid nanoparticles.}

Lipid NPs included solid lipid NPs (SLNs), nanostructured lipid carriers (NLCs), and liposomes. Lipid NPs can be utilized as a vehicle for drug delivery systems [20,21]. They have numerous focal points, contrasted with other colloidal carrier systems like drug entrapment, delayed medication discharge, improved physical and chemical stability, and effective fuse of lipophilic medications in the lipid core of the SLNs and NLCs [22]. Encapsulation of drugs in lipid NPs [23]. Prolonging the medication delivery time in the circulation system diminishes the medicine symptoms and builds its remedial consequences for CNS issues. These attributes make lipid NPs truly valuable for drug delivery to the CNS [24,25].

\subsection{Polymeric micelles.}

Polymeric micelles have been produced for sedate conveyance as of late [26,27]. These micelles structure precipitously in amphiphilic copolymer solution and show shell-core structures, which comprise hydrophobic block polymers (e.g., L, D-lactine polycaprolactone) as a core and hydrophilic square polymer (normally PEGs) as a shell [28]. The polymeric micelles are described to have a molecule size of 10-100 nm. The core could be stacked with a water-insoluble drug. These structures improve drug bioavailability [26]. The micelle shell shields drug from collaborating with serum proteins and non-target cells. The stacked drug discharges through the diffusion mechanism after reaching the target The straightforward structure of the pharmaceutical system with no surface changes is the principal property, making this system valuable for drug delivery to the brain and CNS [29].

\subsection{Dendrimers.}

Dendrimers are atoms with an exceptionally stretched and three-dimensional structure, comprising an underlying core, a few inside layers, redundant units, and a few terminal dynamic surface groups [30]. The parts of dendrimers and surface groups are expanded with upgrading the quantity of dendrimer generation[31]. Dendrimers have low dispersion and superior performance [31,32]. Dendrimers are known as one of the perfect particles with a controlled nanoscale structure for drug delivery $[33,34]$. Dendrimers encourage the vehicle of drugs in different cell membranes or natural boundaries by endocytosis through cell disguise [35,36]. Dendrimers are a potent carrier in intranasal drug delivery [37].

\subsection{Nanoparticles.}

Pharmaceutical NPs have been created to control the drug release and shield it from enzymatic or synthetic debasement to improve its therapeutic efficacy [38,39]. A few sorts of NPs can be utilized to convey drugs and gens to the CNS. The NPs drugs have been incredibly evolved to encourage the productive delivery of insoluble drugs to the CNS to cure neurogenerative disorders [40]. Likewise, the outside of the NPs is regularly adjusted by PEG to upgrade the stability of the colloidal solution and draw out its support in the body [41]. The nanoparticles are a potent carrier for intranasal drug delivery [42], as illustrated in Table 1. 


\section{Neurological Disorders (Parkinson's and Depression)}

Neurodegenerative illnesses (NDs) are problems that occur due to innovative loss of particular important neuronal populations or, in a few instances, related to protein aggregates [43]. The semis folded proteins are chargeable for neuronal degradation and essentially neuronal death. Oxidative stress, generation of loose radicals/reactive oxygen species, mitochondrial dysfunctions, impaired bioenergetics and DNA damage, neuro-inflammatory techniques, and disruption of mobile/axonal transport are linked to the formation of toxic varieties NDP (nucleoid disruption protein)-related proteins. The leading purpose for ND is complicated; however, normally is related to an age-associated decline of neuronal and behavioral functions[44]. Parkinson's and depression are chronic and progressive disorders of CNS.

\subsection{Parkinson's disease.}

Parkinson's disease (PD) is the most widely recognized age-related neurodegenerative disorder with an around-the-world commonness in the millions. The main source of PD is to decrease dopamine levels because of the degradation of dopaminergic neurons in the substantia nigra pars compacta $(\mathrm{SNc})[45]$. This inhibitory abatement impact of dopamine while increments excitatory impact of acetylcholine and at last produce challenges in controlling and commencement of deliberate development of muscle [44]. Side effects are characterized by tremors very still, rigidity, a shortcoming of wilful development, postural precariousness, and freezing. Now, there is evaluated 7 to 10 million individuals worldwide live with Parkinson's disease [46]. The frequency of Parkinson's is increasingly inclined with age; however, an expected $4 \%$ of individuals with PD are analyzed previously at the age of 50. Men are more defenseless than ladies [47] - the spread and scenes of PD increment with age. PD rates are expanding increasingly around worldwide. Neurons are transmitted with the assistance of DA and are fundamental for daily physical movement. In this way, a decline in DA level for neurotransmission in corpus striatum characterized PD and prompts irregular developments [48]. Pathogenesis of PD is very convoluted and observing more than one mechanism like expanding oxidative pressure, an imperfection in mitochondrial capacities, arrangement of plaque-like $\alpha$-synuclein, unevenness of calcium homeostasis, and some hereditary components. These all might be liable for the extreme demise of neurons [49].

\subsection{Current drug therapy by intranasal route for Parkinson's disease.}

The choice of drug treatment is based on different factors like symptoms, age factors, and health issues, also depend on patient needs and stage of PD and metabolism. Different classes of drugs are available for the treatment of PD and classes are cholinesterase inhibitors, dopamine agonists, and monoamine oxidase type B inhibitors but from all of this levodopa is mostly used medication to treat PD [50]. Levodopa is normally utilized, which gives symptomatic relief and creates resilience, and has some unsafe reactions, such as reaction variances and levodopa-initiated dyskinesia(LID) [51]. Because of all these conditions, creating a controlled-release delivery technique is important to lighten the rate of side effects and build a dopamine stimulant effect. Furthermore, researchers are followed by novel methodologies for rewarding PD. Furthermore, among these, the intranasal drug delivery route creates a consideration of specialists for improving a patient's way of life. The following area 
talks about the medications used to treat PD and their patent-related distribution through the intranasal course. The following section talks about the drug used to treat PD and their patentrelated distribution through the intranasal route.

\subsection{Ropinirole.}

Ropinirole following under the class of DA agonist is a non-ergot subsidiary showing high selectivity toward D2 receptors [52]. This kind of DA agonist can be administrated alone or alongside another mix of drugs like levodopa to treat PD [53]. The various formulations are formulated by using nanotechnology. Nanoformulation has the greatest chances for intranasal drug delivery to explain the limitation related to customary ropinirole and increment of uptake of the drug in the brain [54]. Ropinirole has relativity low oral bioavailability due to its extensive hepatic first-pass metabolism. It uses various forms of nanocarrier ropinirole delivered to the brain by intranasal route; one example of ropinirole intranasal drug delivery formulation is spray-dried microparticles by using sodium alginate for ropinirole intranasal delivery [55]. Various kinds of nanoformulation are accessible for intranasal drug delivery of ropinirole.

\subsection{Rotigotine.}

Rotigotine, another class of non-ergoline DA agonists used in treating PD and off-mark treatment of Willis-Ekbom Disease (moreover known as Restless Leg Syndrome) presents agonistic movement on DA receptors giving more inclination to D3 receptor than some other. The mechanism of action of this class of drugs is undetermined however induced to have the capacity to stimulate DA in caudate-putamen, explicitly D2 receptor [56]. A few nano carrierbased drug delivery probes Rotigotine have been led by scientists to upgrade the delivery of Rotigotine to CNS, for example, intranasal approach with micro emulsion-based gel, microspheres, nanoemulsion [57]. For instance, advanced pharmaceutical utilization of nanoemulsion in a mix with intranasal drug delivery approach through mucoadhesive specialist has captivated the interest of many researchers because of the benefits related to fastly drug transport to brain from nasal route [58]. One example of Rotigotine mucoadhesive Nanoemulsion by intranasal route to brain is one alternative approach to solve the oral drug delivery problem [59]. Mucoadhesive nanoemulsion is a good Nano formulation for intranasal drug delivery [60]. Therefore, nanotechnology is a great tool for direct drug delivery to the brain.

\subsection{Pramipexole.}

Pramipexole is a non-ergot derivative that displays natural movement with DA receptors explicitly at the D2 subfamily, indicating greater proclivity towards D3 than different subtypes like D2 or D4 receptors [61]. The activity of Pramipexole being undefined depends on the stimulating capacity of DA receptors inside striatum, which is upheld up by physiologic creature contemplates that clarifies activating of DA receptors at the site of neurons in substantia nigra and striatum [62]. Researchers carried out several studies on Pramipexole. one example of Pramipexole intranasal drug delivery is the preparation of polymeric nanoparticles for brain-to-nose drug delivery; as a result, nanoformulation shows better effect [63]. 


\subsection{Depression.}

Depression is a typical mental disorder, influencing $>300$ million individuals of any age all around, and this number is ascending at a quick pace. Depression is the main source of incapacity worldwide, a significant supporter of the overall global burden of disease. Depression is mostly found in adult age people, but more than $50 \%$ of patients are unable to give a response to the first-line pharmacological treatment of antidepressant drugs because of $\mathrm{BBB}$ [64]. The $\mathrm{BBB}$ is complex that contains cerebral endothelial cells that express efflux transmembrane proteins, mainly from the ATB-binding cassette family, which mainly include P-glycoprotein (P-gp) and breast-cancer-resistant protein (BCRP) [65]. These transporters limit the entrance of lipophilic drugs to the brain, and they have been seen as overexpressed in the brain of hard-headed patients. Because of the good therapy of antidepressant drugs based on drug concentration in the brain, oral and parenteral therapy have limitations because they cannot cross BBB. Therefore, there is a rising requirement for new successful doses form that controllably release of the drug straightforwardly into the brain for decreasing adverse effect, drug interaction, and systemic exposure but intranasal drug delivery proved that have the potential to pass blood-brain barrier by enhancing target ability and decrease systemic adverse effect [66]. The nasal route protects the drug from chemical and metabolic degradation, enhances solubility, drug nasal residence time, and delivers the drug exactly at the point of absorption, bypassing the biological membrane [67]. The following section discusses current intranasal drug therapy for depression.

\section{Current Drug Therapy by Intranasal Route for Depression}

\subsection{Venlafaxine hydrochloride.}

Venlafaxine hydrochloride (VLF) is a double-action antidepressant drug that belongs to the serotonin-norepinephrine reuptake inhibitor (SNRI) pharmacotherapeutic class[68]. VLF is a broadly utilized antidepressant, being commercially accessible as quick and controlledrelease tablets and capsules. VLF is a hydrophilic compound that presents a restricted BBB permeability, short half-life (4-5 h), broad hepatic impact, and low oral bioavailability (45\%), in this way requiring frequent administration to ensure a blood level for therapeutic concentration. Besides, oral treatment gives a moderate beginning of the activity, and clinical symptoms like tachycardia, increase blood pressure, fatigue, cerebral pain, unsteadiness, sexual dysfunction, dry mouth [69], so the nasal route is an alternative to oral administration of different nanoparticles, nanostructured lipid carrier by using chitosan [70] or alginate chitosan nanoparticle [71].

\subsection{Desvenlafaxine.}

Desvenlafaxine (DVF), a second-generation SNRI, was chosen as the drug for the nose to brain delivery. Desvenlafaxine is a functioning metabolite of venlafaxine with an oral bioavailability of $80 \%$ and half plasma existence of $11 \mathrm{~h}$. Even though desvenlafaxine has better serotonin: norepinephrine proportion (10:1) than venlafaxine $(30: 1)$, oral treatment is related to various symptoms, for example, expanded circulatory strain and pulse, constipation, tremor, perspiring, queasiness, migraine, and sleep disturbance [72]. Therefore, nasal formulation for desvenlafaxine delivery is an alternative way for delivery bypassing BBB. 
Various types of nanoparticles are formulated using suitable polymers such as chitosan and polylactide-co-glycolide (PLGA) for the delivery of desvenlafaxine [73].

\subsection{Ketamine and esketamine.}

Ketamine and esketamine is a fast, significant stimulant effect and the plausible against a self-destructive effect that was transient when restricted to single administration [74]. Aside from the clinical results, the inborn difficulties of intravenous (from a coordination's perspective, medical clinic confirmation, and consultation with an anesthesiologist are required) or oral (decreased bioavailability) esketamine administration have provoked its intranasal delivery as a liked and progressively advantageous route for quiet selfadministration. Surely, intranasal esketamine has been accounted for to create a pharmacokinetic profile like that got with higher dosages that were intravenously administrated generally rapid activity and expanded bioavailability of the drug once intranasally administered are inferable from the rich vasculature and moderately high systemic absorption of esketamine by the nasal mucosa [75]. The clinical proof preferring an antidepressant effect and better decency profile related to intranasal esketamine led the FDA to assign this drug an advancement treatment assignment [76]. That justifies the help in the clinical trial in progress with intranasal esketamine since, if the results recovered are sure, approval will be allowed for esketamine nasal splash (Spravato1) new drug application for the treatment of depression in adults. Therefore, it proved that esketamine is a rapid-acting antidepressant.

Table 1. Different Nasal Delivery System for Parkinson's and Depression targeted Drug Delivery.

\begin{tabular}{cccc}
\hline Delivery platform & Incorporated Drug & Target & Source \\
\hline Polymer-Lipid microparticle & Ropinirole & Anti-Parkinson's & {$[77]$} \\
Gel (thermoresponsive/in situ) & Ropinirole & Anti-Parkinson's & {$[78]$} \\
& Venlafaxine & Anti-depressant & {$[79]$} \\
Nanoparticle & & & \\
& Pramipexole dihydrochloride & Anti-Parkinson's & {$[63]$} \\
Microparticle & Desvenlafaxine & Antidepressant & {$[73]$} \\
Niosomes & Resveratrol & Anti-Parkinson's & {$[80]$} \\
\end{tabular}

\section{Conclusion}

Effective treatment of Neurological disorders is limited because of the BBB barrier, so drug delivery to CNS is challenging, but intranasal drug delivery is a novel delivery route that overcomes disturbance by Blood-Brain Barrier and delivers the drug to CNS, and protects the drug from systemic exposure and increases bioavailability. Different types of drugs are delivering to the CNS by using various nanocarriers, which are helpful to treat neurological disorders. It can be concluded that an intranasal route is a non-invasive approach for drug delivery to the brain.

\section{Funding}

This research received no external funding.

\section{Acknowledgments}

This research has no acknowledgment. 


\section{Conflicts of Interest}

\section{The authors declare no conflict of interest.}

\section{References}

1. Barnabas, W. Drug targeting strategies into the brain for treating neurological diseases. Journal of Neuroscience Methods 2019, 311, 133-146,https://doi.org/10.1016/j.jneumeth.2018.10.015.

2. Martins, P.P.; Smyth, H.D.C.; Cui, Z. Strategies to facilitate or block nose-to-brain drug delivery. International Journal of Pharmaceutics 2019, 570,https://doi.org/10.1016/j.ijpharm.2019.118635.

3. Gatto, E.M.; Rojas, N.G.; Persi, G.; Etcheverry, J.L.; Cesarini, M.E.; Perandones, C. Huntington disease: Advances in the understanding of its mechanisms. Clinical Parkinsonism \& Related Disorders 2020, 3, https://doi.org/10.1016/j.prdoa.2020.100056.

4. Williams-Gray, C.H.; Worth, P.F. Parkinson's disease. Medicine 2020, 48, 595-601, https://doi.org/10.1016/j.mpmed.2020.06.001.

5. Deli, M.A. Potential use of tight junction modulators to reversibly open membranous barriers and improve drug delivery. Biochimica et Biophysica Acta (BBA) - Biomembranes 2009, 1788, 892-910, https://doi.org/10.1016/j.bbamem.2008.09.016.

6. Tushar, K.V.; Aliasgar, S.; Sudhanva, M.; Ambikanandan, M. Intranasal Drug Delivery for Brain Targeting. Current Drug Delivery 2005, 2, 165-175,https://doi.org/10.2174/1567201053586047.

7. Gartziandia, O.; Egusquiaguirre, S.P.; Bianco, J.; Pedraz, J.L.; Igartua, M.; Hernandez, R.M.; Préat, V.; Beloqui, A. Nanoparticle transport across in vitro olfactory cell monolayers. International Journal of Pharmaceutics 2016, 499, 81-89,https://doi.org/10.1016/j.ijpharm.2015.12.046.

8. Dufes, C.; Olivier, J.-C.; Gaillard, F.; Gaillard, A.; Couet, W.; Muller, J.-M. Brain delivery of vasoactive intestinal peptide (VIP) following nasal administration to rats. International Journal of Pharmaceutics 2003 , 255, 87-97,https://doi.org/10.1016/S0378-5173(03)00039-5.

9. Crowe, T.P.; Greenlee, M.H.W.; Kanthasamy, A.G.; Hsu, W.H. Mechanism of intranasal drug delivery directly to the brain. Life Sciences 2018, 195, 44-52,https://doi.org/10.1016/j.lfs.2017.12.025.

10. Lochhead, J.J.; Thorne, R.G. Intranasal delivery of biologics to the central nervous system. Advanced Drug Delivery Reviews 2012, 64, 614-628,http://dx.doi.org/10.1016/j.addr.2011.11.002.

11. Mygind, N.; Anggård, A. Anatomy and physiology of the nose--pathophysiologic alterations in allergic rhinitis. Clinical reviews in allergy 1984, 2, 173-188.

12. Smith, T.D.; Bhatnagar, K.P. Anatomy of the olfactory system. In: Handbook of Clin Neurology. Volume 164, 2019;pp. 17-28,https://doi.org/10.1016/B978-0-444-63855-7.00002-2.

13. Mittal, D.; Ali, A.; Md, S.; Baboota, S.; Sahni, J.K.; Ali, J. Insights into direct nose to brain delivery: current status and future perspective. Drug Delivery 2014, 21, 75-86, https://doi.org/10.3109/10717544.2013.838713.

14. Agrawal, M.; Saraf, S.; Saraf, S.; Antimisiaris, S.G.; Chougule, M.B.; Shoyele, S.A.; Alexander, A. Nose-tobrain drug delivery: An update on clinical challenges and progress towards approval of anti-Alzheimer drugs. Journal of Controlled Release 2018, 281, 139-177,https://doi.org/10.1016/j.jconrel.2018.05.011.

15. Johnson, N.J.; Hanson, L.R.; Frey, W.H. Trigeminal Pathways Deliver a Low Molecular Weight Drug from the Nose to the Brain and Orofacial Structures. Molecular Pharmaceutics 2010, 7, 884-893, https://doi.org/10.1021/mp100029t.

16. Samaridou, E.; Alonso, M.J. Nose-to-brain peptide delivery - The potential of nanotechnology. Bioorganic \& Medicinal Chemistry 2018, 26, 2888-2905, https://doi.org/10.1016/j.bmc.2017.11.001.

17. Thorne, R.G.; Pronk, G.J.; Padmanabhan, V.; Frey, W.H. Delivery of insulin-like growth factor-I to the rat brain and spinal cord along olfactory and trigeminal pathways following intranasal administration. Neuroscience 2004, 127, 481-496,https://doi.org/10.1016/j.neuroscience.2004.05.029.

18. Pardeshi, C.V.; Belgamwar, V.S. Direct nose to brain drug delivery via integrated nerve pathways bypassing the blood-brain barrier: an excellent platform for brain targeting. Expert Opinion on Drug Delivery 2013, 10, 957-972,https://doi.org/10.1517/17425247.2013.790887.

19. Nau, R.; Sörgel, F.; Eiffert, H. Penetration of Drugs through the Blood-Cerebrospinal Fluid/Blood-Brain Barrier for Treatment of Central Nervous System Infections. Clinical Microbiology Reviews 2010, 23, 858883,https://doi.org/10.1128/CMR.00007-10.

20. Khezri, K.; Saeedi, M.; Maleki Dizaj, S. Application of nanoparticles in percutaneous delivery of active ingredients in cosmetic preparations. Biomedicine \& Pharmacotherapy 2018, 106, 1499-1505, https://doi.org/10.1016/j.biopha.2018.07.084.

21. Blasi, P.; Giovagnoli, S.; Schoubben, A.; Ricci, M.; Rossi, C. Solid lipid nanoparticles for targeted brain drug delivery. Advanced Drug Delivery Reviews 2007, 59, 454-477,https://doi.org/10.1016/j.addr.2007.04.011.

22. Patel, S.G.; Patel, M.D.; Patel, A.J.; Chougule, M.B.; Choudhury, H. Solid lipid nanoparticles for targeted brain drug delivery. Nanotechnol Targe Drug Deli Sys Brain Tumors2018, 191244,http://dx.doi.org/10.1016/B978-0-12-812218-1.00008-7. 
23. Akbari, J.; Saeedi, M.; Morteza-Semnani, K.; Rostamkalaei, S.S.; Asadi, M.; Asare-Addo, K.; Nokhodchi, A. The design of naproxen solid lipid nanoparticles to target skin layers. Colloids and Surfaces B: Biointerfaces 2016, 145, 626-633,http://dx.doi.org/10.1016/j.colsurfb.2016.05.064.

24. Peira, E.; Marzola, P.; Podio, V.; Aime, S.; Sbarbati, A.; Gasco, M.R. In Vitro and In Vivo Study of Solid Lipid Nanoparticles Loaded with Superparamagnetic Iron Oxide. Journal of Drug Targeting 2003, 11, 1924,https://doi.org/10.1080/1061186031000086108.

25. Gastaldi, L.; Battaglia, L.; Peira, E.; Chirio, D.; Muntoni, E.; Solazzi, I.; Gallarate, M.; Dosio, F. Solid lipid nanoparticles as vehicles of drugs to the brain: Current state of the art. European Journal of Pharmaceutics and Biopharmaceutics 2014, 87, 433-444, https://doi.org/10.1016/j.ejpb.2014.05.004.

26. Zhang, Y,; Huang, Y,; Li, S. Polymeric micelles: Nanocarriers for cancer-targeted drug delivery. AAPS Pharm Sci Tech2014, 15(4), 862-71. https://doi.org/10.1208/s12249-014-0113-z.

27. Kedar, U.; Phutane, P.; Shidhaye, S.; Kadam, V. Advances in polymeric micelles for drug delivery and tumor targeting. Nanomedicine: Nanotechnology, Biology and Medicine 2010, 6, 714-729, http://dx.doi.org/10.1016/j.nano.2010.05.005.

28. Yokoyama, M.; Miyauchi, M.; Yamada, N.; Okano, T.; Sakurai, Y.; Kataoka, K.; Inoue, S. Characterization and anticancer activity of the micelle-forming polymeric anticancer drug adriamycin-conjugated poly(ethylene glycol)-poly(aspartic acid) block copolymer. Cancer research 1990, 50, 1693-1700.

29. Xu, W.; Ling, P.; Zhang, T. Polymeric Micelles, a Promising Drug Delivery System to Enhance Bioavailability of Poorly Water-Soluble Drugs. Journal of Drug Delivery 2013, 2013, 115,https://doi.org/10.1155/2013/340315.

30. Palmerston Mendes, L.; Pan, J.; Torchilin, V.P. Dendrimers as Nanocarriers for Nucleic Acid and Drug Delivery in Cancer Therapy. Molecules 2017, 22, 1-21,https://doi.org/10.3390/molecules22091401.

31. Arrigo, A.; La Ganga, G.; Nastasi, F.; Serroni, S.; Santoro, A.; Santoni, M.-P.; Galletta, M.; Campagna, S.; Puntoriero, F. Artificial, molecular-based light-harvesting antenna systems made of metal dendrimers and multibodipy species. Comptes Rendus Chimie 2017, 20, 209-220, http://dx.doi.org/10.1016/j.crci.2016.02.011.

32. Xu, L.; Zhang, H.; Wu, Y. Dendrimer Advances for the Central Nervous System Delivery of Therapeutics. ACS Chemical Neuroscience 2014, 5, 2-13,https://doi.org/10.1021/cn400182z.

33. Menjoge, A.R.; Kannan, R.M.; Tomalia, D.A. Dendrimer-based drug and imaging conjugates: design considerations for nanomedical applications. Drug Discovery Today 2010, 15, 171-185, http://dx.doi.org/10.1016/j.drudis.2010.01.009.

34. Imae, T. Physicochemical Properties of Dendrimers and Dendrimer Complexes. Dendrimer-Based Drug Deliv Syst From Theory to Pract2012, 55-92,https://doi.org/10.1002/9781118275238.ch2.

35. Xu, L.; Andrew Yeudall, W.; Yang, H. Dendrimer-based RNA interference delivery for cancer therapy. ACS Symp Ser 2013, 1135, 197-213,https://doi.org/10.1021/bk-2013-1135.ch012.

36. Sadekar, S.; Ghandehari, H. Transepithelial transport and toxicity of PAMAM dendrimers: Implications for oral drug delivery. Advanced Drug Delivery Reviews 2012, 64, 571-588, http://dx.doi.org/10.1016/j.addr.2011.09.010.

37. Ke, W.; Shao, K.; Huang, R.; Han, L.; Liu, Y.; Li, J.; Kuang, Y.; Ye, L.; Lou, J.; Jiang, C. Gene delivery targeted to the brain using an Angiopep-conjugated polyethyleneglycol-modified polyamidoamine dendrimer. Biomaterials 2009, 30, 6976-6985,http://dx.doi.org/10.1016/j.biomaterials.2009.08.049.

38. Maleki Dizaj, S.; Lotfipour, F.; Barzegar-Jalali, M.; Zarrintan, M.-H.; Adibkia, K. Physicochemical characterization and antimicrobial evaluation of gentamicin-loaded $\mathrm{CaCO} 3$ nanoparticles prepared via microemulsion method. Journal of Drug Delivery Science and Technology 2016, 35, 1623,http://dx.doi.org/10.1016/j.jddst.2016.05.004.

39. Maleki Dizaj, S.; Lotfipour, F.; Barzegar-Jalali, M.; Zarrintan, M.-H.; Adibkia, K. Ciprofloxacin HCl-loaded calcium carbonate nanoparticles: preparation, solid state characterization, and evaluation of antimicrobial effect against Staphylococcus aureus. Artificial Cells, Nanomedicine, and Biotechnology 2017, 45, 535-543, https://doi.org/10.3109/21691401.2016.1161637.

40. Yadav, A.; Ghune, M.; Kumar Jain, D. Nano-medicine based drug delivery system. J Adv Pharm Educ Res 2011, 1, 201-13.

41. Singh, R.; Lillard, J.W. Nanoparticle-based targeted drug delivery. Experimental and Molecular Pathology 2009, 86, 215-223,http://dx.doi.org/10.1016/j.yexmp.2008.12.004

42. Asha Spandana, K.M.; Bhaskaran, M.; Karri, V.V.S.N.R.; Natarajan, J. A comprehensive review of nano drug delivery system in the treatment of CNS disorders. Journal of Drug Delivery Science and Technology 2020, 57,https://doi.org/10.1016/j.jddst.2020.101628.

43. Quek, C.; Hill, A.F. The role of extracellular vesicles in neurodegenerative diseases. Biochemical and Biophysical Research Communications 2017, 483, 1178-1186,http://dx.doi.org/10.1016/j.bbrc.2016.09.090.

44. Singh, D.; Kapahi, H.; Rashid, M.; Prakash, A.; Majeed, A.B.A.; Mishra, N. Recent prospective of surface engineered Nanoparticles in the management of Neurodegenerative disorders. Artificial Cells, Nanomedicine, and Biotechnology 2016, 44, 780-791,https://doi.org/10.3109/21691401.2015.1029622. 
45. Rastegari, E.; Azizian, S.; Ali, H. Machine Learning and Similarity Network Approaches to Support Automatic Classification of Parkinson's Diseases Using Accelerometer-based Gait Analysis. Proc 52 ${ }^{\text {nd }}$ Hawaii Int Conf Syst Sci2019, 6, 4231-42,https://doi.org/10.24251/HICSS.2019.511.

46. Paul, A.; Yadav, K.S. Parkinson's disease: Current drug therapy and unraveling the prospects of nanoparticles. Journal of Drug Delivery Science and Technology 2020, 58,https://doi.org/10.1016/j.jddst.2020.101790.

47. Huang, P.; Goetz, C.G.; Woolson, R.F.; Tilley, B.; Kerr, D.; Palesch, Y.; Elm, J.; Ravina, B.; Bergmann, K.J.; Kieburtz, K. Using global statistical tests in long-term Parkinson's disease clinical trials. Movement Disorders 2009, 24, 1732-1739, https://doi.org/10.1002/mds.22645.

48. Surmeier, D.J.; Ding, J.; Day, M.; Wang, Z.; Shen, W. D1 and D2 dopamine-receptor modulation of striatal glutamatergic signaling in striatal medium spiny neurons. Trends in Neurosciences 2007, 30, 228-235, https://doi.org/10.1016/j.tins.2007.03.008.

49. Patel, A.; Surti, N.; Mahajan, A. Intranasal drug delivery: Novel delivery route for effective management of neurological disorders. Journal of Drug Delivery Science and Technology 2019, 52, 130-137, https://doi.org/10.1016/j.jddst.2019.04.017.

50. Aquilonius, S.-M.; Nyholm, D. Development of new levodopa treatment strategies in Parkinson's diseasefrom bedside to bench to bedside. Upsala Journal of Medical Sciences 2017, 122, 71-77, http://dx.doi.org/10.1080/03009734.2017.1285374.

51. Tran, T.N.; Vo, T.N.N.; Frei, K.; Truong, D.D. Levodopa-induced dyskinesia: clinical features, incidence, and risk factors. Journal of Neural Transmission 2018, 125, 1109-1117,http://dx.doi.org/10.1007/s00702018-1900-6.

52. Klewe, I.V.; Nielsen, S.M.; Tarpø, L.; Urizar, E.; Dipace, C.; Javitch, J.A.; Gether, U.; Egebjerg, J.; Christensen, K.V. Recruitment of $\beta$-arrestin2 to the dopamine D2 receptor: Insights into anti-psychotic and anti-parkinsonian drug receptor signaling. Neuropharmacology 2008, 54, 1215-1222, https://doi.org/10.1016/j.neuropharm.2008.03.015.

53. Malewar, N.; Avachat, M.; Pokharkar, V.; Kulkarni, S. Controlled Release of Ropinirole Hydrochloride from a Multiple Barrier Layer Tablet Dosage Form: Effect of Polymer Type on Pharmacokinetics and IVIVC. AAPS PharmSciTech 2013, 14, 1178-1189,https://doi.org/10.1208/s12249-013-0009-3.

54. Torres-Ortega, P.V.; Saludas, L.; Hanafy, A.S.; Garbayo, E.; Blanco-Prieto, M.J. Micro- and nanotechnology approaches to improve Parkinson's disease therapy. Journal of Controlled Release 2019, 295, 201-213, https://doi.org/10.1016/j.jconrel.2018.12.036.

55. Hussein, N.; Omer, H.; Ismael, A.; Albed Alhnan, M.; Elhissi, A.; Ahmed, W. Spray-dried alginate microparticles for potential intranasal delivery of ropinirole hydrochloride: development, characterization and histopathological evaluation. Pharmaceutical Development and Technology 2020, 25, 290-299, https://doi.org/10.1080/10837450.2019.1567762.

56. LeWitt, P.A.; Lyons, K.E.; Pahwa, R. Advanced Parkinson disease treated with rotigotine transdermal system. Neurology 2007, 68, 1262-1267,https://doi.org/10.1212/01.wnl.0000259516.61938.bb.

57. Hira, C.; Bapi, G.; Bappaditya, C.; Uttam, K.M.; Pinaki, S.; Rakesh, K.T. Pharmacokinetic and Pharmacodynamic Features of Nanoemulsion Following Oral, Intravenous, Topical and Nasal Route. Current Pharmaceutical Design 2017, 23, 2504-2531,https://doi.org/10.2174/1381612822666161201143600.

58. Nirale, P.; Paul, A.; Yadav, K.S. Nanoemulsions for targeting the neurodegenerative diseases: Alzheimer's, Parkinson's and Prion's. Life Sciences 2020, 245,https://doi.org/10.1016/j.lfs.2020.117394.

59. Choudhury, H.; Zakaria, N.F.B.; Tilang, P.A.B.; Tzeyung, A.S.; Pandey, M.; Chatterjee, B.; Alhakamy, N.A.; Bhattamishra, S.K.; Kesharwani, P.; Gorain, B.; Md, S. Formulation development and evaluation of rotigotine mucoadhesive nanoemulsion for intranasal delivery. Journal of Drug Delivery Science and Technology 2019, 54,https://doi.org/10.1016/j.jddst.2019.101301.

60. Bonferoni, M.C.; Rossi, S.; Sandri, G.; Ferrari, F.; Gavini, E.; Rassu, G.; Giunchedi, P. Nanoemulsions for "Nose-to-Brain" Drug Delivery. $\quad$ Pharmaceutics $\quad 2019, \quad 11, \quad 1-$ 17,https://doi.org/10.3390/pharmaceutics11020084.

61. Perez-Lloret, S.; Rey, M.V.; Ratti, L.; Rascol, O. Pramipexole for the treatment of early Parkinson's disease. Expert Review of Neurotherapeutics 2011, 11, 925-935, https://doi.org/10.1586/ern.11.75.

62. Hubble, J.P.; Koller, W.C.; Cutler, N.R.; Sramek, J.J.; Friedman, J.; Goetz, C.; Ranhosky, A.; Korts, D.; Elvin, A. Pramipexole in Patients with Early Parkinson's Disease. Clinical Neuropharmacology 1995, 18, 338-347,https://doi.org/10.1097/00002826-199508000-00006.

63. Raj, R.; Wairkar, S.; Sridhar, V.; Gaud, R. Pramipexole dihydrochloride loaded chitosan nanoparticles for nose to brain delivery: Development, characterization and in vivo anti-Parkinson activity. International Journal of Biological Macromolecules 2018, 109, 27-35,http://dx.doi.org/10.1016/j.ijbiomac.2017.12.056.

64. O’Leary, O.F.; Dinan, T.G.; Cryan, J.F. Faster, better, stronger: Towards new antidepressant therapeutic $\begin{array}{llllll}\text { strategies. } \quad \text { European Journal of } & \text { Pharmacology }\end{array}$ http://dx.doi.org/10.1016/j.ejphar.2014.07.046.

65. Bicker, J.; Alves, G.; Fortuna, A.; Falcão, A. Blood-brain barrier models and their relevance for a successful development of CNS drug delivery systems: A review. European Journal of Pharmaceutics and Biopharmaceutics 2014, 87, 409-432,http://dx.doi.org/10.1016/j.ejpb.2014.03.012. 
66. Fan, Y.; Chen, M.; Zhang, J.; Maincent, P.; Xia, X.; Wu, W. Updated Progress of Nanocarrier-Based Intranasal Drug Delivery Systems for Treatment of Brain Diseases. 2018, 35, 433-467, https://doi.org/10.1615/CritRevTherDrugCarrierSyst.2018024697.

67. Moinuddin, S.G.A.; Razvi, S.H.; Uddin, M.; Fazil, M.; Shahidulla, S.; Akmal, M.J.T.P.I.J. Nasal drug delivery system: A innovative approach. Pharma Innov J2019, 8, 169-77.

68. Banzi, R.; Cusi, C.; Randazzo, C.; Sterzi, R.; Tedesco, D.; Moja, L. Selective serotonin reuptake inhibitors (SSRIs) and serotonin-norepinephrine reuptake inhibitors (SNRIs) for the prevention of migraine in adults. The Cochrane database of systematic reviews 2015, 4.

69. Vitorino, C.; Silva, S.; Bicker, J.; Falcão, A.; Fortuna, A. Antidepressants and nose-to-brain delivery: drivers, restraints, opportunities and challenges. Drug Discovery Today 2019, 24, 1911-1923, https://doi.org/10.1016/j.drudis.2019.06.001.

70. Haque, S.; Md, S.; Fazil, M.; Kumar, M.; Sahni, J.K.; Ali, J.; Baboota, S. Venlafaxine loaded chitosan NPs for brain targeting: Pharmacokinetic and pharmacodynamic evaluation. Carbohydrate Polymers 2012, 89, 72-79,http://dx.doi.org/10.1016/j.carbpol.2012.02.051.

71. Haque, S.; Md, S.; Sahni, J.K.; Ali, J.; Baboota, S. Development and evaluation of brain targeted intranasal alginate nanoparticles for treatment of depression. Journal of Psychiatric Research 2014, 48, 1-12, http://dx.doi.org/10.1016/j.jpsychires.2013.10.011.

72. Perry, R.; Cassagnol, M. Desvenlafaxine: A new serotonin-norepinephrine reuptake inhibitor for the treatment of adults with major depressive disorder. Clinical Therapeutics 2009, 31, 1374-1404, http://dx.doi.org/10.1016/j.clinthera.2009.07.012.

73. Tong, G.-F.; Qin, N.; Sun, L.-W. Development and evaluation ofDesvenlafaxine loaded PLGA-chitosan nanoparticles for brain delivery. Saudi Pharmaceutical Journal 2017, 25, 844-851, http://dx.doi.org/10.1016/j.jsps.2016.12.003.

74. Molero, P.; Ramos-Quiroga, J.A.; Martin-Santos, R.; Calvo-Sánchez, E.; Gutiérrez-Rojas, L.; Meana, J.J. Antidepressant Efficacy and Tolerability of Ketamine and Esketamine: A Critical Review. CNS Drugs 2018, 32, 411-420,https://doi.org/10.1007/s40263-018-0519-3.

75. Morrison, R.L.; Fedgchin, M.; Singh, J.; Van Gerven, J.; Zuiker, R.; Lim, K.S.; van der Ark, P.; Wajs, E.; Xi, L.; Zannikos, P.; Drevets, W.C. Effect of intranasal esketamine on cognitive functioning in healthy participants: a randomized, double-blind, placebo-controlled study. Psychopharmacology 2018, 235, 11071119,https://doi.org/10.1007/s00213-018-4828-5.

76. Ionescu, D.F.; Papakostas, G.I. Experimental medication treatment approaches for depression. Translational Psychiatry 2017, 7, e1068-e1068, 1-8,http://dx.doi.org/10.1038/tp.2017.33.

77. Karavasili, C.; Bouropoulos, N.; Sygellou, L.; Amanatiadou, E.P.; Vizirianakis, I.S.; Fatouros, D.G. PLGA/DPPC/trimethylchitosan spray-dried microparticles for the nasal delivery of ropinirole hydrochloride: in vitro, ex vivo and cytocompatibility assessment. Materials Science and Engineering: C 2016, 59, 10531062, http://dx.doi.org/10.1016/j.msec.2015.11.028.

78. Rao, M.; Agrawal, D.K.; Shirsath, C. Thermoreversible mucoadhesive in situ nasal gel for treatment of Parkinson's disease. Drug Development and Industrial Pharmacy 2017, 43, 142-150, https://doi.org/10.1080/03639045.2016.1225754.

79. Bhandwalkar, M.J.; Avachat, A.M. Thermoreversible Nasal In situ Gel of Venlafaxine Hydrochloride: Formulation, Characterization, and Pharmacodynamic Evaluation. AAPS PharmSciTech 2013, 14, 101-110, https://doi.org/10.1208/s12249-012-9893-1.

80. Trotta, V.; Pavan, B.; Ferraro, L.; Beggiato, S.; Traini, D.; Des Reis, L.G.; Scalia, S.; Dalpiaz, A. Brain targeting of resveratrol by nasal administration of chitosan-coated lipid microparticles. European Journal of Pharmaceutics and Biopharmaceutics 2018, 127, 250-259,https://doi.org/10.1016/j.ejpb.2018.02.010.

81. Sita, V.G.; Jadhav, D.; Vavia, P. Niosomes for nose-to-brain delivery of bromocriptine: Formulation development, efficacy evaluation and toxicity profiling. Journal of Drug Delivery Science and Technology 2020, 58,https://doi.org/10.1016/j.jddst.2020.101791. 